\title{
Linx
}

Revue des linguistes de l'université Paris X Nanterre

42 | 2000

Approches sociolinguistiques du plan phonique

\section{De la variation et des variantes : à propos du relâchement}

\section{Bernard Laks}

\section{(2) OpenEdition}

Journals

Édition électronique

URL : http://journals.openedition.org/linx/747

DOI : $10.4000 / \operatorname{linx} .747$

ISSN : 2118-9692

Éditeur

Presses universitaires de Paris Nanterre

\section{Édition imprimée}

Date de publication : 1 juin 2000

Pagination : $21-28$

ISSN : 0246-8743

\section{Référence électronique}

Bernard Laks, « De la variation et des variantes : à propos du relâchement », Linx [En ligne], 42 | 2000, mis en ligne le 20 juin 2012, consulté le 30 avril 2019. URL : http://journals.openedition.org/linx/747 ; DOI : $10.4000 / \operatorname{linx} .747$

Ce document a été généré automatiquement le 30 avril 2019.

Département de Sciences du langage, Université Paris Ouest 


\title{
De la variation et des variantes : à propos du relâchement
}

\author{
Bernard Laks
}

\section{Linguistique variationniste ${ }^{1}$}

1 Historiquement issue du courant sociolinguistique labovien, la linguistique variationniste est, dès son origine, marquée par une ambition théorique et modélisatrice qui dépasse de loin les frontières acceptées de la sociolinguistique. En effet, si la sociolinguistique prend pour objet les modalités de la variation externe (variations sociales, stylistiques, géographiques, diachroniques etc.) et la façon dont ces variations se trouvent investies de valeur socio-différentielle, la linguistique variationniste quant à elle prend pour objet les modèles linguistiques et la théorie des grammaires dont elle interroge la capacité à rendre compte théoriquement de ces dimensions de la variation (Cf. Labov (1972)).

Deux types d'objets, deux niveaux d'analyse très différents, deux concepts sont ainsi distingués. La variation comme dimension structurelle interne d'une part, la valeur sociodifférentielle d'autre part. Il s'ensuit une autonomie de principe de la valeur sociale des variantes par rapport à leur définition structurale. Dans cette approche, les variantes observées en un temps, en un lieu et dans une formation sociale donnés, constituent donc en quelque sorte les points de cristallisation de ces deux dimensions abstraites et autonomes. Cette distinction conceptuelle entre variation (inhérente) et investissement social des variantes est particulièrement utile lorsqu'on aborde la question du « relâchement » phonique.

\section{Des variantes}

Comme j'ai tenté de le montrer ailleurs (Laks (1992)), la linguistique variationniste se définit d'abord comme une méthode et c'est au plan empirique qu'elle rencontre tout d'abord la variation des usages. Cette variation externe est spécifiquement l'objet de la sociolinguistique. Pour nous en tenir au plan phonique, les modifications d'aperture ou 
d'antériorité, les amuïssements de tous ordres, les syncopes, apocopes, aphérèses, synérèses et diérèses, les déplacements d'accent, bref tout ce qui affecte la forme phonique et produit un écart par rapport à une norme, toujours hypothétique et pourtant socialement prégnante, toutes ces dimensions de variation donc, ne sont pas socialement neutres et inertes. Elles ont une efficace sociale qu'il importe d'analyser. La sociologie est alors convoquée.

Pour ne prendre qu'une seule référence sociologique, une théorie structurale de l'organisation topologique du champ social comme celle de Bourdieu, par exemple (Bourdieu (1979), Bourdieu (1994)), permet de comprendre comment ces dimensions de la variation des usages se trouvent investies de valeurs socio-différentielles. L'accumulation en des points précis du champ social d'usages linguistiques particuliers et leur association régulière avec des sujets socialement qualifiés pourvus d'autres espèces de capitaux (social, économique, culturel etc.), finit par précipiter et cristalliser ce qu'il est possible d'appeler un capital linguistique: au-delà de la maîtrise de tels parlers ou de tels styles (parler populaire, parler jeune, parler soutenu, style oratoire etc.), une instance qui renvoie à l'aisance du locuteur à se mouvoir dans l'espace sociolinguistique, à son sens du placement et à sa capacité à adapter son style linguistique au niveau exigé par l'organisation sociale de la situation de parole.

5 Du point de vue sociologique, c'est donc l'association régulière entre usages linguistiques spécifiques et sujets socialement spécifiés qui définit la valeur des variantes. Dans cette analyse, la valeur socio-différentielle, comme toute autre valeur sociale, est donc entièrement relative. Elle est un sous-produit de l'organisation du champ social et n'existe pas en dehors de cette organisation positionnelle. On y reconnaîtra une réinterprétation sociologique de la loi de la valeur et de la formation des prix des économistes, notamment marxistes. C'est l'accumulation et la concentration de biens en certains points de l'espace social, et leur raréfaction concomitante en d'autres points, qui crée le marché et en régule les échanges par une loi de formation des prix.

6 Tout l'intérêt linguistique de cette analyse sociologique de la valeur des variantes consiste donc à rappeler que la valeur socio-différentielle ne doit rien à la nature des variantes en jeu mais doit tout à la structure du marché social sur lequel elles sont placées. Ce n'est pas parce que telle variante est linguistiquement meilleure qu'elle est sociolinguistiquement classante. Tout au contraire, c'est parce qu'elle fait partie, à un moment donné du temps, des usages de locuteurs socialement classés qu'elle en arrive à être perçue comme un des principes de leur classement.

7 Pour illustrer cette loi de formation des prix et l'autonomie de la loi de la valeur sociodifférentielle par rapport au système linguistique lui-même, il suffit de considérer la façon dont la valeur d'une même variante fluctue avec le temps et avec la formation sociale. Sans qu'il soit nécessaire de revenir ici sur l'analyse sociolinguistique de la chute des liquides post-consonantiques en position finale (Laks (1977); Laks (1980); Laks (1983)), on admettra que les prononciations [liv], [fnct], [kat] pour livre, fenêtre, quatre sont profondément déclassantes, et marquent aujourd'hui les styles les plus populaires et les moins surveillés. Pourtant, il n'en a pas toujours été ainsi. On sait que les liquides et spécialement $/ \mathrm{r} /$ constituent depuis fort longtemps une zone d'instabilité du système phonologique du français ${ }^{2}$ et qu'au cours du temps, la valeur socio-différentielle de leur chute a beaucoup changé. Dauzat (1967) note par exemple que cette syncope alors extrêmement classante est attestée dans le style le plus soutenu, celui de la poésie de Ronsard ou de Marguerite de Navarre ${ }^{3}$. Si au $17^{\text {ème }}$ siècle elle n'est plus très valorisée, 
Bourciez (1950) note encore qu'au " $18^{\text {ème }}$ siècle, les formes quat(re), not(re), vo(tre) étaient admises pour les proclitiques même dans la conversation polie » (p. 185). Au début du $19^{\text {ème }}$ cette syncope constitue un véritable stéréotype social pour la nouvelle aristocratie du Directoire. Enfin, on sait qu'aujourd'hui, l'assourdissement et l'amuïssement sont de règle pour tous les styles à la pause et que l'articulation des liquides, lorsqu'elle est maintenue et dans les styles les plus surveillés, y est, au mieux, chuchotée (Grammont (1914) ; Nyrop (1934)).

Au cours du temps la même variable s'est donc trouvée investie de valeurs sociodifférentielles différentes, sinon antithétiques. Le comportement de cette zone d'instabilité structurale du phonétisme français illustre ainsi parfaitement notre propos et souligne l'autonomie de la loi de la valeur. En effet, si la même variable a pu être assignée aussi bien aux styles les plus surveillés qu'aux parlers les plus populaires c'est bien que la valeur socio-diffrérentielle de cette variable n'est pas liée à la variable ellemême. Sa valeur est entièrement extrinsèque au système linguistique et ne dépend que de la loi de formation des prix à un moment donné, sur un marché linguistique donné.

Pour autant, l'attribution de valeur socio-différentielle à des usages différents suppose l'existence d'une instabilité structurelle du système. Pour qu'il y ait variante, il faut que la plasticité du système phonologique ouvre la possibilité de variation. C'est le cas en français pour les liquides depuis cinq siècles au moins. Pour en comprendre la raison et pour analyser les mécanismes internes de cette variation, il faut alors abandonner l'interface sociolinguistique et se tourner du côté du système phonologique. L'analyse linguistique interne est alors convoquée.

\section{De la variation}

10 La linguistique variationniste modélise la plasticité structurale des systèmes linguistiques en ayant recours au concept de variation inhérente. C'est une propriété interne de tout système linguistique que d'user de formes phonétiques légèrement différentes pour construire un même contenu sémantique de message. L'existence de variantes d'usage découle ainsi directement de l'hétérogénéité structurale qui marque tout système linguistique. On sait que cette variation interne au système n'est pas libre. Elle est contrainte par le système lui-même et limitée dans son ampleur par les nécessités de l'intercompréhension. Dans des langues comme le français où la pression normative est constante et où la norme orthographique entrave les dynamiques de changements en cours, les zones de variation inhérente bougent peu : liaison, élision, e muet, $\mathrm{h}$ aspiré, aperture vocalique, liquides et glissantes constituent depuis plusieurs siècles les lieux de variation inhérente où s'enracinent les usages socialement différenciés des mêmes variables.

11 Ainsi, si l'on en croit le témoignage de John Palsgrave (Palsgrave (1530)), depuis le $16^{\text {ème }}$ siècle au moins, la prononciation ou la syncope de certaines consonnes finales en contexte de liaison est l'objet d'une grande variation. Si en français contemporain il n'existe aucun locuteur, quel que soit son style de parole ou son profil social, qui ne lie l'article pluriel sur le nom (des $\frown$ oranges), personne en revanche ne lie toujours certaines locutions variables (mais $\cap$ en effet, pas $\cap$ encore). La tri-partition des contextes de liaison en liaisons obligatoires (catégoriques), variables et interdites (impossibles) rend compte du fait que, bien que les frontières en aient changé, il existe aujourd'hui comme hier une zone d'hétérogénéité et de variation inhérente pour ce qui concerne la liaison. L'analyse 
structurale que l'on peut en faire (Cf. par exemple Encrevé (1988)) est entièrement interne. Elle met fondamentalement en jeu l'instabilité des consonnes finales en français et le fait que le gabarit des unités morphologiques est, lexicalement, partiellement sous-spécifié. La forme particulière qu'ont les représentations phonologiques en français pour ce qui concerne la fin des mots explique et motive les sandhis externes qui marquent notre langue. L'interaction des processus syllabiques, des processus d'ancrage et des conditions de liage, induit une grande variabilité potentielle dans l'actualisation de ces sandhis. C'est la liaison variable telle que nous pouvons l'observer en surface. On remarquera au passage que cette variation n'est aucunement libre. Définie par le système, elle est contrainte et limitée par les quelques latitudes qu'il permet.

12 Ce trop bref rappel de l'analyse structurale de la variation inhérente de la liaison illustre à nouveau notre propos. L'analyse de la variation inhérente, l'analyse des facteurs structuraux qui conditionnent la variation, est totalement muette quant à l'évaluation sociale des variantes qui apparaîtront en surface. En effet, ce que la linguistique variationniste permet d'expliquer, ce n'est pas la valeur des variantes, c'est leur existence même comme supports potentiels de valeur socio-différentielle. Les deux plans d'analyse, linguistique interne et sociolinguistique des usages sont donc bien, en droit comme en fait, distincts.

\section{Du relâchement}

13 Cette séparation nette qui construit la linguistique variationniste comme une linguistique des modèles et la sociolinguistique (variationniste) comme une science de l'interface entre système linguistique hétérogène et marché linguistique socialement structuré possède de nombreuses vertus, entre autres prophylactiques. Elle interdit en particulier de rechercher dans l'ordre de la langue ce qui relève du jeu du marché linguistique et de l'enjeu des distinctions sociales.

La littérature descriptive d'inspiration sociolinguistique, et surtout dialectologique, fait assez souvent un usage très peu formalisé du terme « relâchement ». De ce terme, comme de celui de «loi du moindre effort » avec lequel il commute parfois, on peut faire deux lectures très différentes. Une lecture phonologique qui y lit le relâchement de la tension articulatoire, et une lecture sociologique plus directement péjorative. Dans les usages non contrôlés, c'est toujours, volens nolens, la péjoration sociale qui l'emporte.

Considérons tout d'abord le plan sociologique. Dans la ligne de notre analyse précédente qui renvoie l'évaluation des variantes à la seule loi sociologique de construction de la valeur, on pourrait, pour reprendre un argumentaire bien connu de Bourdieu, montrer que le relâchement articulatoire dont il est ici question ne procède que de l'homologie structurale qui tend à s'établir entre qualification sociale et hexis corporelle; bref, que les sujets sociaux qui ne doivent leur position de dominés qu'à « un fâcheux relâchement de la volonté et de la morale " adoptent par définition un style relâché dans toutes leurs attitudes et toutes leurs actions. A contrario du dicton c'est donc bien l'homme (socialement qualifié) qui qualifie le style.

16 Même dans les usages les plus contrôlés, ce contenu sociologique péjoratif n'est jamais totalement absent de la notion de relâchement. C'est pourquoi ce terme doit être phonologiquement critiqué et déconstruit. Notons tout d'abord que le relâchement de la tension articulatoire dans certaines positions correspond à un changement diachronique 
dans toute la Romania, changement extrêmement ancien et durable. Ce changement trouve pour une part sa source dans la perte de la quantité et la libération de l'accent dès le latin vulgaire. Dans la diachronie du français, l'amuïssement en position finale qui fait chuter les consonnes correspond à un changement par le haut, socialement très classant, qui n'a aucun rapport avec le contenu péjoratif du terme relâchement.

17 A l'inverse de la lecture sociologique, on peut noter que le changement actuel qui affecte la prononciation des consonnes finales dites fixes (dix mai, un but, un fait, un mas) est un changement par le bas qui correspond au rétablissement d'une consonne que la norme tient ici pour muette. Tout le contraire donc d'un relâchement. De même ne peut-on pas tenir pour articulatoirement relâché, un usage vernaculaire qui allonge et diérèse la finale de certain verbes (que je [swajə], qu'il [عjə], qu'il [krwajə]). Par rapport à une finale sans coda, cette prononciation ne relâche pas mais renforce la structure syllabique. Certes, on pourra arguer de la perte de la gémination (intellectuel, excellent, allusif) pour stipendier le relâchement de ceux qui ne parlent pas comme ils devraient écrire, mais si la perte du redoublement raccourcit le geste articulatoire, on ne voit pas comment la consonne latérale résiduelle pourrait être relâchée. Elle reste, comme toujours en français, tendue.

Le critère de tension, qui implique on l'a compris une critique implicite de la notion de relâchement, est particulièrement intéressant pour ce qui concerne les voyelles. En effet, rappelons que toutes les voyelles $\mathrm{du}$ français contemporain, en tous styles, sont toujours tendues, c'est à dire articulées avec une très forte tension musculaire et une importante décharge d'énergie. C'est même une des caractéristiques les plus tranchées de notre système vocalique. Sauf pour ce qui concerne le comportement de schwa, il n'y a donc aucun sens à parler en français de relâchement vocalique au sens d'affaiblissement lié à un plus faible contrôle. Et puisque nous parlons du seul amuïssement vocalique réellement productif, celui qui conduit à schwa et à sa syncope, notons que le maintien de nombreux schwas, notamment en position finale (une [tablə :], [madamə :], une [krEə :]), n'est pas l'un des traits les moins singuliers des parlers les plus vernaculaires. Bien que la chute de segments soit toujours identifiée avec le relâchement (social) et leur maintien avec l'excellence, rappelons avec Martinon (1913) que pour ce qui concerne les schwas des post-clitiques qui ne peuvent plus chuter, l'usage le plus normatif et le plus classant était au $17^{\text {ème }}$ de les relâcher jusqu'à les syncoper totalement. Molière écrit par exemple : "Eh mon petit Monsieur, prenez-l' d'un peu moins haut ».

Considérons enfin les systèmes vocaliques à deux /a/, dont l'un nettement vélaire. On sait qu'ils marquaient avant guerre les parlers les plus ouvriers. Georges Marchais avait encore ainsi des [a] parfaitement vélaires devant liquides vélaires ([kapitat [animał). Le relâchement de la tension articulatoire ne saurait être invoqué pour ces parlers si l'on se souvient que par rapport aux articulations centrales et moyennes, le [a] vélaire suppose une beaucoup plus grande énergie articulatoire (ouverture maximale, abaissement et rétraction de la racine). Le même argument peut être construit à partir de l'analyse du style articulatoire d'un certain nombre de jeunes issus de l'émigration, style marqué par de nombreuses pharyngalisations et glottalisations, qui fort loin de relâcher l'énergie articulatoire, rendent la production sonore beaucoup plus tendue, complexe et donc musculairement coûteuse.

Ainsi, un survol rapide de quelques variations actuellement actives en français permet de déconstruire l'homologie entre relâchement articulatoire et péjoration sociale. De nombreuses variantes produites par relâchement de tension furent, et sont, classantes. A 
l'inverse, un grand nombre de traits attribués aux parlers les plus vernaculaires ou les plus populaires procèdent non d'un relâchement, mais d'une complexification articulatoire, d'une augmentation de la tension, ou d'un accroissement du coût musculaire. On voit à nouveau que l'analyse structurale interne des variantes et leur évaluation sociale sur le marché linguistique sont deux dimensions nettement distinctes. La naturalisation des différences sociolinguistiques impliquées par l'usage ambigu du terme relâchement doit être combattue, et les homologies qui structurent notre vision sociale des usages linguistiques doivent être critiquées et déconstruites.

\section{BIBLIOGRAPHIE}

BOURCIEZ, Edouard (1950). Petit précis historique de phonétique française. Paris, Klincksieck

BOURDIEU, Pierre (1979). La distinction : critique sociale du jugement. Paris, Éditions de Minuit

BOURDIEU, Pierre (1994). Raisons pratiques : sur la théorie de l'action. Paris, Seuil

DAUZAT, Alfred (1967). Phonétique et grammaire historique de la langue française. Paris

ENCREVÉ, Pierre (1988). La liaison avec et sans enchaînements : phonologie tridimentionnelle et usages du français. Paris, Le Seuil

FouchÉ, Pierre (1959). Traité de prononciation française. Paris, Klincksieck

GADET, Françoise (2000). « Note sur le relâchement comme terme sociolinguistique. » Linx 42 :

11-20.

Grammont, Maurice (1914). Traité pratique de prononciation française. Paris, Delagrave

LABOV, William (1972). Language in the Inner City: Studies in the Black English Vernacular, Philadelphia, University of Pennsylvania Press (trad. fr. (1978), Le parler ordinaire. Paris, Editions de Minuit).

LAKS, Bernard (1977). « Contribution empirique à l'analyse socio-différentielle de la chute de /r/ dans les groupes consonantiques finals. » Langue Française 34 : 109-25.

LAKS, Bernard (1980). Différenciation linguistique et différenciation sociale : quelques problèmes de sociolinguistique française. Thèse. Université de Paris VIII

LAKS, Bernard (1983). « Langage et pratiques sociales : étude sociolinguistique d'un groupe d'adolescents. » Actes de la recherche en sciences sociales 46 : 73-97.

LAKS, Bernard (1992). « La linguistique variationniste comme méthode. » Langages 108 : 34-51.

MAlmberg, Bertil (1974). Manuel de phonétique générale. Paris, Picard

MARTINON, Philippe (1913). Comment on prononce le français. Traité complet de prononciation pratique avec les noms propres et les noms étrangers. Paris, Larousse

NYRoP, Kristoffer (1934). Manuel de phonétique du français parlé. Copenhague,Trad. Fr. Paris ; Picard NYROP, Kristoffer (1950). Grammaire historique de la langue française. Copenhague, Glydendal 
PALSGRAVE, John (1530). Lesclarcissement de la langue française. Londres

WACHS, Sandrine (1997). Le relâchement de la prononciation en français parlé de l'Ile-de-France. Analyse linguistique et sociolinguistique par générations. Thèse. Paris X Nanterre

\section{NOTES}

1. Cet article doit beaucoup à la lecture de Wachs (1997) et surtout à celle de l'article de Françoise Gadet dans ce numéro (Gadet (2000)). Il ne s'agit pas à proprement parler d'une réponse, puisque on verra que je suis d'accord avec la ligne générale de l'argumentaire présenté par Gadet, mais d'une élaboration sur le terrain phonologique à partir des pistes qu'elle ouvre.

2. Fouché (1959) note une tendance à l'amuïssement des liquides, spécialement finales, dès l'époque impériale dans l'est de la Romania. On sait également qu'aux $15^{\text {ème }}, 16^{\text {ème }}$ et $17^{\text {ème }}$ siècles toute une série de changements, parfois opposés, affectent $/ \mathrm{r} /:$ postériorisation, rhotacisme, syncope dans les désinences verbales, remotivation lexicale, etc. Pour Malmberg (1974), la postériorisation est un changement par le haut initié par la grande bourgeoisie commerçante des villes du nord.

3. $C f$. également Nyrop (1950)

\section{RÉSUMÉS}

En phonologie comme en sociolinguistique, la notion de relâchement est l'une des moins précises qui soit. S'appuyant sur une relecture de la linguistique variationniste, et de la sociologie des biens symboliques, cet article propose une critique empirique et théorique de cette notion. On montre comment les variations historiques dans l'évaluation des variantes tout comme l'analyse phonologique de la complexité des formes conduisent à abandonner cette notion.

Either in sociolinguistics or in phonology, "relachement" is not a clear and usefull notion. Building on the results of variationist linguistics and on the sociology of symbolic goods, this papers proposes an empirical and theoretical approach to this problematic notion. Historical changes in the evaluation of forms along with internal analysis of the complexity of uterrances lead to disregard this notion

\section{AUTEUR}

\section{BERNARD LAKS}

Université de Paris X et GDR 1954

Laks@u-paris10.fr 